\title{
IC-PCR1000 Control Using a Wireless Sensor Network (WSN)
}

\author{
M. Bakkali, C. Mascareñas-Perez-Iñigo, and R. Carmona-Galan
}

\begin{abstract}
We put forward in this paper a new method based on a Wireless Sensor Network from Crossbow technology for the IC-PCR1000 control and communication to lessen the impact of the unwanted EMI. This method is a new method that allows working in the band LF/LW/MW/FM without the EMI emitted by the power supply of the PC and IC-PCR1000
\end{abstract}

Index Terms-Wireless Sensor Network (WSN), ICPCR1000, Electromagnetic Interference (EMI)

\section{INTRODUCTION}

The electrical wires can transport electromagnetic signals interferences [1] and play the role of an Electromagnetic Interference (henceforth EMI) antenna [2]. The PC power supply is an important source of EMI, and its internals/externals wires have a function of such EMI antennas. Among them, those coming out of communication port and the sound card.

The IC-PCR 1000 is a wideband radio receiver that is controlled by means of the serial port [3]. It is intended to receive command from the PC RS232 serial port. The effect of EMI stated above bars the reception in some frequency mode [4], when using the IC-PCR1000 connected to the PC [5]. For example, Low Frequency or LF (signals that range from $30 \mathrm{kHz}$ to $300 \mathrm{kHz}$ ), Long Wave or LW (signals that range from $150 \mathrm{kHz}$ to $280 \mathrm{kHz}$ ), and Medium Wave or MW (signals that range from $530 \mathrm{kHz}$ to $1620 \mathrm{kHz}$ ).

We put forward in this paper a new method for the ICPCR1000 control and communication without connecting them to the PC to lessen the impact of the unwanted EMI. In addition to this significance, the system achieved allows some advantages like:

1) Avoiding electromagnetic radiation hazards: ICPCR1000 can be used to take measurement of the signal strength by using the available S-meter function. This can be done in the case of having equipment that has electromagnetic radiation hazards such as radar.

2) Remote control :

- The relevant information in terms of signal measured (level, time, mode, frequency, etc.) are sent from the IC-PCR 1000 to a remote PC through the Wireless Sensor Network (WSN).

M. Bakkali is with Departamento Control y Comunicaciones, División Air Thermodynamics, CIAT. medcasem@hotmail.com mbakkali@grupociat.es

C. Mascareñas-Perez-Iñigo is with Departamento de CC y TT de la Navegación y Teoría de Señal y Comunicación, Universidad de Cádiz.

R. Carmona-Galan is with Instituto de Microelectrónica de Sevilla, IMSE-CSIC, Sevilla.
- The IC-PCR1000 can receive command from a remote PC like setting the frequency, mode, bandwidth filter, volume, squelch, etc.

3) Measurement process Monitoring: When detecting some signal of importance or high concentration, the motes send a message by the air to the base station. This message can be configured to activate an alarm, send an email and/or display data on the PC-base station screen. Additionally, data is saved into a data base which keeps saved all the information about the reception during all the period of scanning for their further processing.
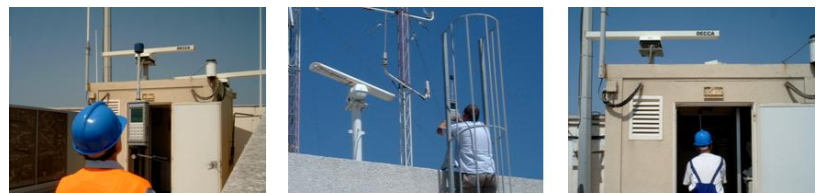

Fig. 1. Example of measurement process made by the research group "naval communications systems and signals"

The method presented in this paper to control the ICPCR1000 is based on a Wireless Sensor Network from Crossbow technology [6] designed for the development of an infrastructure meant for the control and communication of the different system we are using. These motes, which are autonomous, small, robust and versatile systems, are linked to the IC-PCR1000 constituting a powerful and clean-method for the control and communication with ICPCR1000.

In order to establish the necessary interconnection between the radio subsystem and the wireless communications link, we have tested the different ports available at each device [7].

Communication between the different elements of the system is supported by the available UARTs and GPIOs.

\section{ICOM-PCR1000}

The IC-PCR-1000 is a wideband radio receiver that is controlled via the $\mathrm{PC}$ serial port. It has a wideband coverage from $100 \mathrm{kHz}$ to $1.3 \mathrm{GHz}$ and can operate at CW, LSB, USB, AM, FM and WFM mode. The filter bandwidth can be specified for each mode as follows: $F M=6 / 15 / 50 \mathrm{kHz}$, $\mathrm{AM}=3 / 6 / 15 / 50 \mathrm{kHz}, \mathrm{WFM}=50 / 230 \mathrm{kHz}, \mathrm{SSB}=3 / 6 \mathrm{kHz}$ and $\mathrm{CW}=3 / 6 \mathrm{kHz}$.

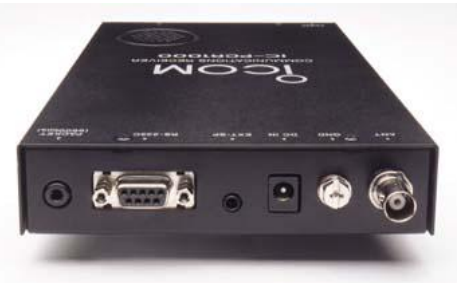

Fig. 2. IC-PCR1000 receiver 
The receiver offer 21 choices for the steep size ranging from $1 \mathrm{~Hz}$ to $1 \mathrm{MHz}$, and have the different following function:

1) The IF shift function is used for SSB and CW mode tuning. The purpose is to modify the pass band with which signals are received. If two signals are quite close and interfering with each other due to limitation in term of narrow band, the IF shift is used to move the pass band away from the offending signal. This helps to reduce or remove the interference. The range for the IF shift is +/$1,2 \mathrm{kHz}$.

2) The AFC function (Automatic Frequency Control) is used to correct signal drift when listening to FM signals. It must be used with a filter of $15 \mathrm{kHz}$ or less to allow a tracking of the core frequency using $100 \mathrm{~Hz}$ steep.

3) The tone squelch function is an FM feature that allows the selection of one of the fifty one tones between 67,0 and 254, $1 \mathrm{~Hz}$.

4) The AGC (Automatic Gain Control) function is used with the CW, SSB and AM modes to set the unstable audio levels due to changes in signal strength.

5) The NB (Noise Blanker) function is used to eliminate pulse-type noise from SSB, CW and AM signals.

6) The VSC (Voice Scan Control) function is used to help skip over uncontrolled carriers as well as birdie-type frequencies.

\section{WIRELESS COMMUNICATION SUPPORT}

The communication used between the different systems is supported by an IRIS XM2110 mote [6, 7]. This mote features $250 \mathrm{Kbp}$ data rate, and outdoor line-of-sight ranges as far as $500 \mathrm{~m}$ between nodes without amplification. The selection of a commercial platform is mainly based on the availability, together with a matured and tested hardware of a wide set of software tools. To be precise, an operating system, a programming language and compiler [8] for the mote microprocessor (Atmega1281), a software environment for mote programming and network monitoring and application development (Mote Works).

\section{A. Mote-PCR1000 Communication}

The most direct way to communicate the IC-PCR1000 with the mote is using the available UARTs. Several adjustments are required. First, the serial port of the XM2110 mote operates by default at a higher baud rate than the IC-PCR1000; consequently, data that interchanges between them is initially disabled. This can be overcome by changing the mote baud rate at the software level.

Another difficulty found is in the voltage levels representing the different logic states. An adapter chip is employed for this (MAX3222).

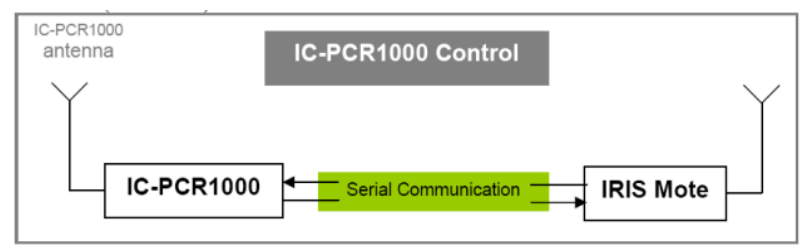

Fig. 3. IC-PCR1000 Control
This node has the following functions:

1) Generates a repetitive sequence of command to be sent to the IC-PCR1000

2) Sends the data over the UART port at every time fired (we use $1000 \mathrm{~ms}$ ) to node 2

3) Constructs the packet filling up the message buffer with data received from IC-PCR1000 UART.

4) Specifies target

5) Broadcasts the message over the air

The next diagram shows the connection for the TinyOS interfaces used.

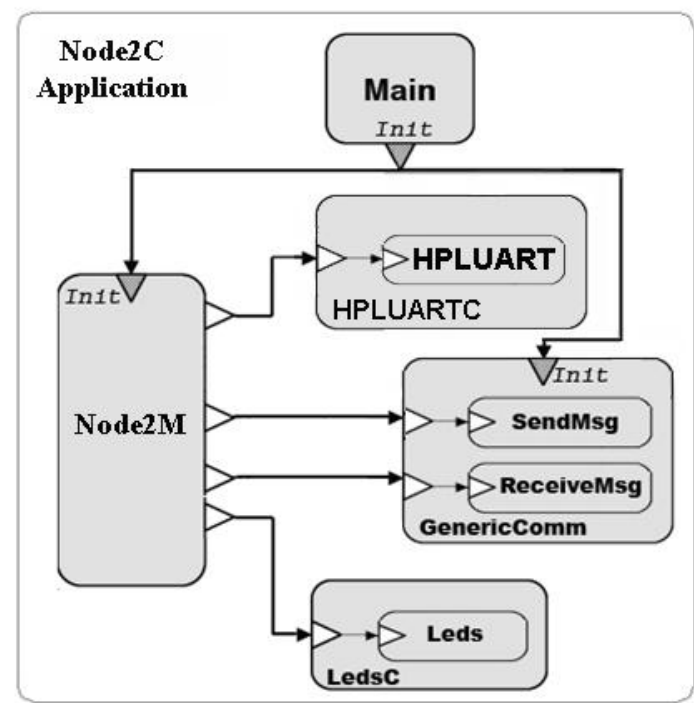

Fig. 4. connection for the interfaces used to program the node connected with the IC-PCR1000.

We have used the HPLUARTC component that is responsible of data transmission over the UART. It provides the interface HPLUSART Control and HPLUART.

HPLUARTC is a low level component that allows sending directly data of one byte over the serial port.

The transmission of data over the UART port is controlled by the component TimerC.

We have used the interfaces SendMsg provided by the GenericComm component to assume the task of sending message.

\section{B. Mote-PC Communication}

This mote is connected to the PC via the UART port and is used to receive the broadcast signal from the mote and then shows the signals on the PC screen through the UART port.

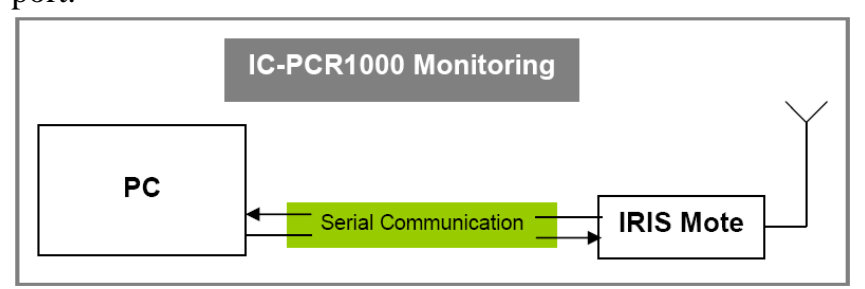

Fig. 5. IC-PCR1000 Monitoring

\section{APPLICATIONS EXAMPLE: IC-PCR1000 REMOTE CONTROL FOR RADAR MEASUREMENT PROCESS}

In this experiment we used two motes. The first one connected to the IC-PCR1000 through the UART port and 
the second is connected to a remote PC through the UART.

The commands are sent from the PC to the mote by the air, and the response from IC-PCR1000 is replied by the same way through the mote connected to this later.

The objective of this experiment is to perform an automatic scanning by the IC-PCR1000 making a sweep over frequencies and modes of interest, so as to evaluate the radar signals behaviour.

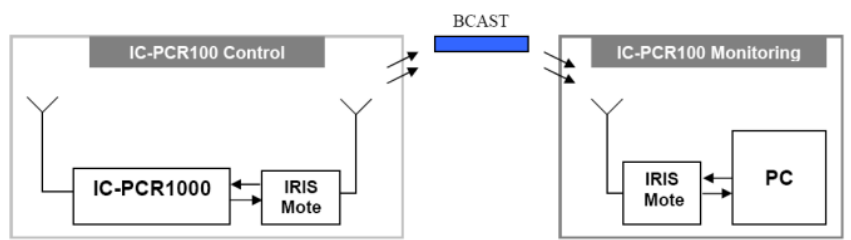

Fig. 6. Applications Example: IC-PCR1000 remote control for Radar measurement process

\section{A. Mote-PCR1000 Programming}

The commands from the mote to the IC-PCR1000 are sent in ASCII format as follow:

$$
\begin{aligned}
& \text { Command }+\mathrm{CR}+\mathrm{LF} \\
& \mathrm{CR}=\mathrm{Chr} \$(13) \\
& \mathrm{LF}=\mathrm{Chr} \$(10)
\end{aligned}
$$

The serial port of the XM2110 operates by default at a baud rate of $115200 \mathrm{bps}$, while the IC-PCR1000's maximum baud rate is $38400 \mathrm{bps}$. Therefore, the baud rate of the XM2110 should be modified. This can be done at the mote software level. The lowest baud rate that is supported by the mote is 1200 bps. We decided to set both systems to 9600 bps.

We set the mote to this speed by changing the function of the file: "HPLUART0M.nc" as follow:

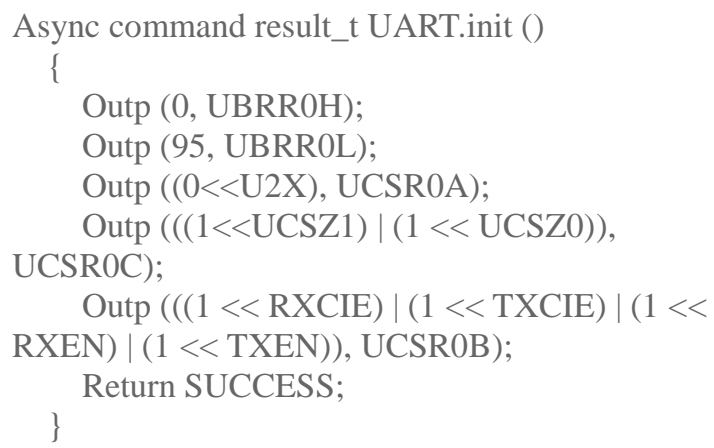

At the IC-PCR1000 stage, the command used to change the baud rate was "G1". To set it to $9600 \mathrm{bps}$, we used the command:

\section{G1 $03\langle\mathrm{CR}\rangle\langle\mathrm{LF}\rangle$}

After changing the baud rate of the receiver it was necessary to use the "G0?" command to check if the connection status was $\mathrm{OK}$.

\section{CONCLUSION}

A new method for controlling the IC-PCR1000 is developed. It is demonstrated that it is possible to communicate with the receiver without the use of the PC. This is achieved by implementing a protocol of communication using an IRIS XM2110 mote from Crossbow. The mote, autonomous, small, robust and versatile systems, is linked to the IC-PCR1000 UART port constituting a powerful and clean-method for the control and communication with IC-PCR1000.

- This method is a new method that allows working in the band LF/LW/MW/FM without the EMI emitted by the power supply of the PC and ICPCR1000.

The experiment made validates the feasibility of the method, and shows applications that can be derived from this, like:

- IC-PCR1000 Remote control

- Avoiding the electromagnetic radiation hazards

- Monitoring of the Measurement process

This method can have an important reference value for amateur radio experiment and measurement made in the LF/LW/MW/FM mode based on the IC-PCR1000.

\section{REFERENCES}

[1] M. Bakkali, C. Mascareñas, and ALL, "Feasibility Study of Advancing and Sitting up Power Line Communication (PLC) System under Circumstances of Electromagnetic Compatibility (EMC) on the Ships," presented at 9th Internacional Conference. Electrical Power Quality and Utilisation, 9-11 de octubre de 2007, Barcelona, Spain.

[2] C. Mascareñas, M. Bakkali, and ALL, "Sistemas de Comunicaciones a través de la Red Eléctrica. Efecto Interferencias PLC en los unifamiliares," presented at International Science and Technology Conference, 21-22 March 2007, Malaga, Spain- 23 March 2007, Tangiers, Morocco.

[3] Icomamerica. [Online]. Available: http://www.icomamerica.com/en/receivers/

[4] C. Mascareñas Y. Pérez-Íñigo, M. Básico de Sistemas de Comunicaciones Marítimas, Universidad de Cádiz

[5] Icom PCR-1000 Hardware controller. [Online]. Available: http://www.astro.hr/ucionica/tom/hardware/

[6] Xbow. [Online]. Available: http:// www.xbow.com

[7] M. Bakkali, R. Carmona, and A. Rodriguez, "A Prototype Node for Wireless Vision Sensor Network Applications Development," International Symposium On Image/Video Communications over fixed and mobile networks ISIVC 2010, 30 de septiembre, 1-2 de octubre de 2010, Rabat, Morocco.

[8] P. Levis, TinyOS/nesC Programming Reference Manual, Crossbow Inc. January 2006.

[9] Russ' ICOM PCR-1000. [Online]. Available: http://gladden.org/pcr1000/index.html

[10] KB9JJA ICOM PCR-1000. [Online]. Available: http://www.wentztech.com/radio/Equipment/PCR1000/PCR1000.htm 1

[11] Strong Signals-ICOM IC-PCR1000 Review. [Online]. Available: http://www.strongsignals.net/access/content/rr_ic-pcr.html

[12] ICOM PCR 1000 Resource. [Online]. Available: http:// http://qsy.to/pcr/

[13] A stand-alone interface that emulates a PC control program. [Online]. Available : http://jetvision.de/pcr1000.shtml 